\title{
LA THÉOLOGIE DES SACREMENTS COMME THÉOLOGIE FONDAMENTALE
}

\section{Bruce T Morrill}

Institut Catholique de Paris | «Transversalités »

2009/3 N 111 | pages 159 à 162

ISSN 1286-9449

Article disponible en ligne à l'adresse :

http://www.cairn.info/revue-transversalites-2009-3-page-159.htm

\section{!Pour citer cet article :}

Bruce T Morrill, «La théologie des sacrements comme théologie fondamentale », Transversalités 2009/3 ( $\left.\mathrm{N}^{\circ} 111\right)$, p. 159-162.

DOI 10.3917/trans.111.0159

Distribution électronique Cairn.info pour Institut Catholique de Paris.

(C) Institut Catholique de Paris. Tous droits réservés pour tous pays.

La reproduction ou représentation de cet article, notamment par photocopie, n'est autorisée que dans les limites des conditions générales d'utilisation du site ou, le cas échéant, des conditions générales de la licence souscrite par votre établissement. Toute autre reproduction ou représentation, en tout ou partie, sous quelque forme et de quelque manière que ce soit, est interdite sauf accord préalable et écrit de l'éditeur, en dehors des cas prévus par la législation en vigueur en France. Il est précisé que son stockage dans une base de données est également interdit. 


\title{
LA THÉOLOGIE DES SACREMENTS COMME THÉOLOGIE FONDAMENTALE
}

\author{
Bruce T. MORRILL, s.j. \\ Professeur, Boston College
}

Les théologiens de la liturgie aiment rappeler que la lex orandi, la loi de la prière, est la lex credendi, la règle de ce qu'il faut croire. Ils ne cessent pourtant de débattre du sens exact que peut prendre dans la vie de l'Église ce principe patristique, issu de la fameuse sentence de Prosper d'Aquitaine. Tout au long de sa prodigieuse carrière universitaire et pastorale, Louis-Marie Chauvet n'a cessé de se demander pourquoi et en quoi la liturgie sacramentelle est essentielle à la foi chrétienne. Les liturgistes tentent souvent de répondre à cette question en s'appuyant sur l'histoire et sur le contenu des rites chrétiens. Chauvet, quant à lui, les envisage à partir de leur nature irréductiblement rituelle, dans une perspective de théologie fondamentale.

La théologie fondamentale s'efforce de penser la révélation divine telle que la transmettent l'Écriture et la tradition, ainsi que les conditions qui disposent l'être humain à la recevoir. Quoique hautement théorique, l'œuvre de Chauvet se réfère constamment aux pratiques liturgiques, usant de nombreux exemples pastoraux. Son ampleur et sa beauté tiennent à plusieurs insistances:

- sur le caractère incarné de notre Rédemption en Christ,

- sur la particularité de cette parole divine que l'Esprit ne cesse d'inscrire dans le corps universel de l'Église à travers l'activité rituelle des sacrements,

- sur l'impératif éthique inhérent à la donation que Dieu fait de luimême, 
- sur l'enracinement de cette éthique sacramentelle dans la révélation biblique du Dieu crucifié.

La célébration des sacrements ne vise pas à gagner un lieu sacré où nous aurions à contempler Dieu par amour de lui, mais à révéler la puissance transformatrice du Dieu de Jésus jusque dans l'ambiguïté de notre vie éthique ${ }^{1}$. La foi trinitaire n'a ici rien d'une abstraction théologique: elle est au cœur d'une foi incarnée. Croire au Dieu crucifié est un scandale pour le théisme (et pour son Dieu monolithique). Le Christ qui vient à nous dans le sacrement est celui qui s'est rendu solidaire de l'homme jusqu'à la mort, nous révélant ainsi la différence de Dieu: source de notre salut, il vient contrecarrer les dieux trop humains de notre imaginaire et établir une similitude entre Dieu et l'homme. Dans le mystère pascal, l'amour entre le Père et le Fils devient amour entre Dieu et l'humanité. L'Esprit est la différence, la sainteté, l'altérité de l'amour partagé entre le Père et le Fils. L'Esprit a inscrit cette différence dans le corps-personne de Jésus de Nazareth en l'élevant de la mort à la gloire, et, à travers les rites de l'Église, il inscrit désormais cette différence dans le corps des fidèles. Dans la célébration du mystère pascal, «Dieu se révèle ainsi comme celui qui "se barre" par l'Esprit dans l'humanité, donnant à cette dernière la possibilité d'advenir comme le "lieu sacramentel" où il continue à prendre corps. $»^{2}$

Si le caractère paradoxal de cette théologie fondamentale frappe l'auditeur, cela tient simplement à ce qu'elle parvient à dire la beauté tragique du mystère pascal: la révélation divine du salut comme rencontre du désir divin et du désir humain dans la personne humaine de Jésus: corporelle et historique, achevée et encore en lutte, défaite mais triomphante. Chaque fois que, par un court-circuit imaginaire, nous croyons pouvoir accéder à la plénitude de la présence divine, nous affadissons la foi, nous déplaçons l'espérance, nous blessons l'amour venu à nous dans l'Esprit du Christ mort et ressuscité. La tradition de l'Église, c'est l'assentiment donné à cet ordre symbolique particulier, au mouvement continuel de vivification mutuelle de la parole, du sacrement et de l'éthique, qui vient nourrir la foi chrétienne. Quand le divorce entre le symbole et la réalité (entre le corps et l'esprit) se

1. «En d'autres termes, tout sacrement nous donne symboliquement à voir et à vivre ce qui fait de notre existence humaine une existence proprement chrétienne » (L.M. Chauvet, Les Sacrements..., p. 161).

2. Ibid., p. 183. 
glisse dans la pratique chrétienne, nous perdons la grâce singulière, la faveur divine que nous offre l'Évangile. Au lieu de nous donner d'avoir part à la grâce que seul Dieu peut conférer dans sa différence salvatrice, la liturgie n'est plus qu'une pauvre expression de ce que nous savons déjà, et les sacrements se réduisent à une dimension quelconque de l'expérience humaine. Les conséquences pastorales peuvent se révéler tragiquement pitoyables, la liturgie sacramentelle de l'Église se trouvant coincée dans un no man's land devenu étranger au déroulement de la vie quotidienne, personnelle, ecclésiale, sociale, économique et politique ${ }^{3}$.

À l'aube du nouveau millénaire, c'est la mission de l'Église qu'il importe de penser, tant ad intra que ad extra. Chrétiens, nous assistons aujourd'hui à un déplacement anthropologique aussi considérable que celui du Moyen Âge, à une profonde transformation (matérielle, intellectuelle, institutionnelle, psychologique) de la façon d'envisager l'humanité. Bien sûr, l'apparence, l'ordre, la vie intellectuelle du christianisme du XIV' siècle rompaient déjà avec les pratiques et les théologies de la fin du premier millénaire ${ }^{4}$. Les conséquences locales et globales des Lumières et de la modernité (scientifique, technologique, économique, politique) se déploient à un rythme tellement accéléré que cela exige des théologiens chrétiens une fidélité accrue à l'Écriture et à la tradition, mais aussi un immense respect du public - ecclésiastique, universitaire ou commun - qu'ils entendent servir 5 .

Les directeurs de cet ouvrage et les auteurs ont mobilisé tous leurs talents non seulement pour rendre hommage à la personne et à l'œuvre monumentale de Louis-Marie Chauvet, mais aussi pour se mettre au

3. Sur ma propre tentative pour affronter ce problème, voir B. T. MoRrILL, Anamnesis as Dangerous Memory: Political and Liturgical Theology in Dialogue, Collegeville, Liturgical Press, 2000.

4. La bibliographie en ce domaine est immense. Je ne mentionnerai ici l'apport que de la seule Carolyn Walker Bynum. Sur les ouvrages établissant la transformation médiévale de la théologie et de la pratique sacramentelle, voir G. MACY, Treasures from the Storeroom: Medieval Religion and the Eucharist, Collegeville, Liturgical Press, 1999. Ma propre pensée à cet égard doit beaucoup aux contributions des historiens de l'Église médiévale lors de la conférence interdisciplinaire que j'ai codirigée au College of the Holy Cross (Worcester, Massachusetts) en 2002. Voir B. T. MorriLl et al. (éd.), Practicing Catholic: Ritual, Body and Contestation in Catholic Faith, New York, Palgrave Macmillan, 2006.

5. Pour une discussion originale entre les théologiens et ces publics, voir D. TRACY, The Analogical Imagination: Christian Theology and the Culture of Pluralism, New York, Crossroad, 1981. 
service de l'Église en permettant quelques avancées novatrices pour la liturgie et les sacrements selon six axes de recherches: théologie fondamentale, théologie biblique, ecclésiologie, théologie morale, sciences sociales et anthropologie théologique. C'est évidemment au lecteur de juger si nos efforts académiques sont à la hauteur de la gratitude personnelle et professionnelle que nous devons à notre collègue LouisMarie Chauvet.

Bruce T. MORRILL, s.j. 